Research Article

\title{
Full-Thickness Skin Graft according to Surrounding Relaxed Skin Tension Line Improves Scar Quality in Facial Defect Coverage: A Retrospective Comparative Study
}

\author{
Jeonghwan Shin, ${ }^{1}$ Uk Jang, ${ }^{2}$ Sang Oon Baek, ${ }^{2}$ and Jun Yong Lee $\mathbb{D}^{2}$ \\ ${ }^{1}$ Daesung Medical Center, Republic of Korea \\ ${ }^{2}$ Department of Plastic and Reconstructive Surgery, College of Medicine, The Catholic University of Korea, Seoul, Republic of Korea \\ Correspondence should be addressed to Jun Yong Lee; ps.jylee@catholic.ac.kr
}

Received 6 June 2021; Accepted 12 August 2021; Published 16 September 2021

Academic Editor: Fabiano Bini

Copyright (c) 2021 Jeonghwan Shin et al. This is an open access article distributed under the Creative Commons Attribution License, which permits unrestricted use, distribution, and reproduction in any medium, provided the original work is properly cited.

\begin{abstract}
A full-thickness skin graft (FTSG) is useful for covering small skin and soft tissue defects. In this paper, we suggest FTSG in consideration of the relaxed skin tension line (RSTL) concept for scar quality improvement since FTSG has disadvantages, including contour irregularities and mismatches of color and texture. We conducted a retrospective chart review of twenty-one patients with skin cancer on the face who underwent wide excision and FTSG by a single surgeon from October 2013 to July 2019. Twenty-one patients with skin cancer on the face were divided into RSTL-matched and RSTL-unmatched groups, and FTSG was performed. Each group was subjected to scar assessment three months after surgery. Observer assessment was performed by five independent observers using the observer component of the patient and observer scar assessment scale (POSAS) and Vancouver scar scale (VSS). Our results indicate that there were significant differences between the RSTL-matched and RSTL-unmatched groups in the VSS and POSAS components. In addition, the RSTL-matched group showed a natural appearance with surrounding tissue in the dynamic animation phase compared to the unmatched group. RSTL-matched FTSG can be an attractive option for face skin and soft tissue defect coverage. (An earlier version of this paper has been presented at the International Conference on PRS Korea 2020.)
\end{abstract}

\section{Introduction}

Skin grafting is the gold standard for covering large-area skin defects when there are partial-thickness defects with intact underlying musculatures [1]. A skin graft can be performed faster than a local flap, and it has the advantage that it has less donor site morbidity and can be repeatedly implemented. However, there are functional and esthetic disadvantages such as wound contracture and color mismatch in skin grafts [2]. Full-thickness skin grafts (FTSG) have been developed to overcome these limitations. When the subdermal fat layer was removed and skin harvesting performed with a thickness of $0.6 \mathrm{~mm}$ or more, contracture was significantly reduced compared to the thinly harvested skin graft, and a significant esthetic outcome was guaranteed by providing thickness and skin appendage to the recipient site [3-5]. Moreover, to overcome the mismatch of texture and color, full-thickness skin was harvested through the donor site adjacent to the defect area [6]. Nevertheless, the mismatch of the skin texture around the grafts and defects made scarring of the graft margins stand out and unnatural results when the graft was taken.

When making an incision in the skin, the operator applies the incision considering the relaxed skin tension line (RSTL). This action ensures cosmetic and functional results by securing minimal tension when closing defects [7]. With this in mind, we thought there would be significant esthetic results when the RSTL of the FTSG was placed parallel to the RSTL of the recipient site.

The main purposes of the present retrospective comparative study are to demonstrate the esthetic outcome and 

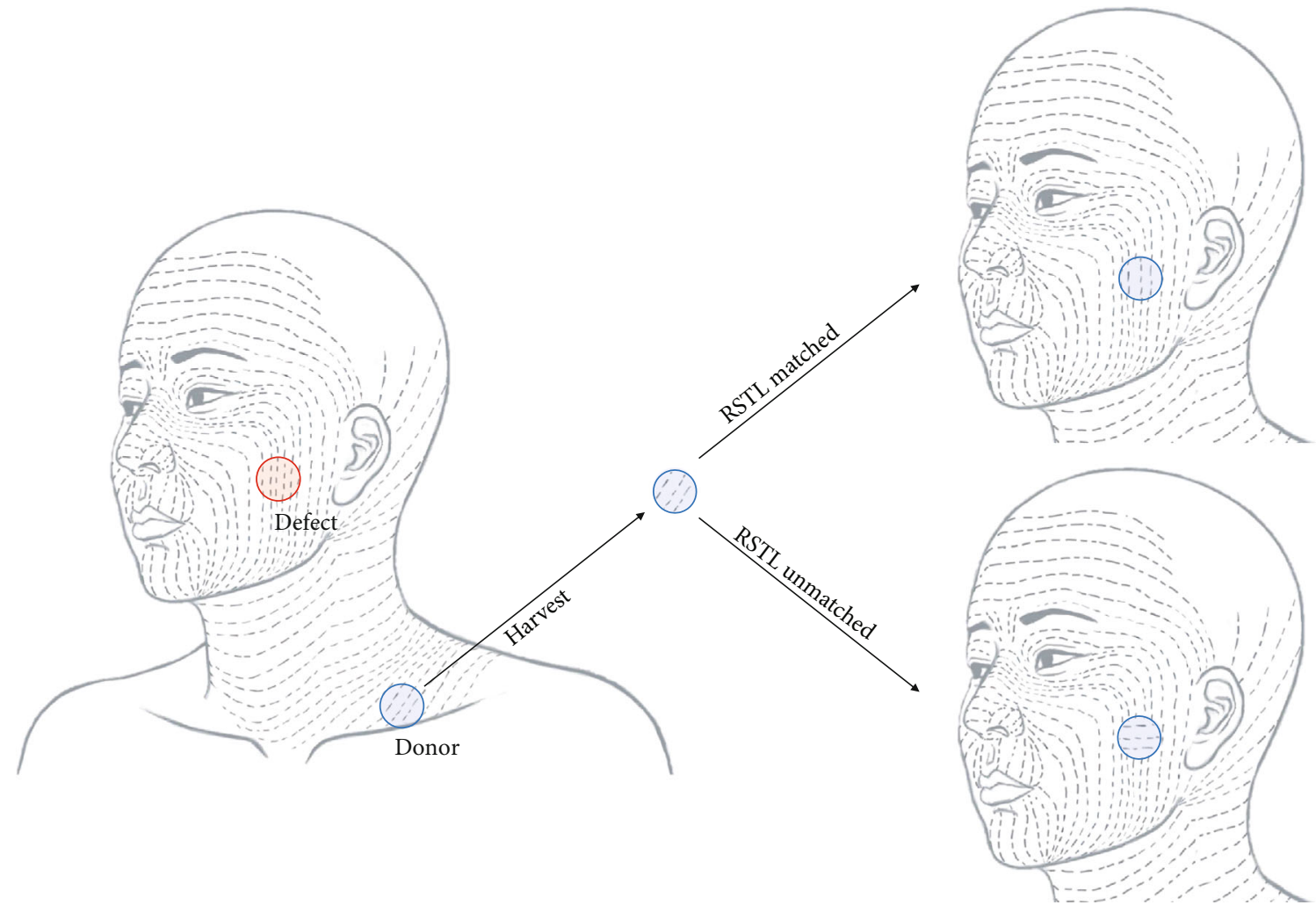

FIGURE 1: The schematic illustration of study design. We performed a wide excision of facial skin cancer and immediately performed a FTSG on the facial skin defect. Patients were divided into groups that underwent FTSG matching RSTL between donor and recipient and those who did not follow RSTL. In the RSTL-matched group, the graft was harvested at the donor site, trimmed according to the defect, and applied to facial defects in accordance with RSTL. In the RSTL-unmatched group, the harvested graft was applied to the facial defect regardless of the RSTL of the donor graft.

satisfaction of skin graft through RSTL matching from the perspective of patients and observers.

\section{Patients and Methods}

The Catholic Medical Center institutional review board approved our study (IRB OC20RASI0108). A retrospective chart review of twenty-one patients who underwent wide excision of facial skin cancer and immediately underwent a FTSG on the facial skin defect was conducted at the Catholic Medical Center in Korea over a 7-year period (2013-2019).

We performed a wide excision of facial skin cancer and immediately performed a FTSG on the facial skin defect. Patients were divided into groups that underwent FTSG matching RSTL between donor and recipient and those who did not follow RSTL (Figure 1). In the RSTL-matched group, the graft was harvested at the donor site, trimmed according to the defect, and applied to facial defects in accordance with RSTL. In the RSTL-unmatched group, the harvested graft was applied to the facial defect regardless of the RSTL of the donor graft. Finally, the graft was fixed in a tie-over manner using \#5-0 black silk. The donor site was closed primarily. A scar assessment of the patient and the observer was performed three months after the operation. The scar was evaluated using two types of assessment tools. Results were analyzed according to the assessment tool, observer, and patient. Thereafter, we tried to determine the difference in scar assessment between patients and observers by comparing the RSTL-matched and RSTLunmatched groups.

The decision to match RSTL was made intraoperatively and by the attending plastic surgeon. Since the surgical outcome of FTSG may vary by surgical technique, infection, complications, and so on, all surgeries were performed by a single senior surgeon (JY Lee) to prevent these problems, and there was no difference in the procedure in the two groups except for the grafted skin's RSTL orientation. In addition, as a problem occurred in grafts taken, cases requiring secondary procedures such as debridement were not included in this study.

Data on patient age, sex, defect area, defect site, donor site, and type of skin cancer were obtained. Each patient was surveyed for patient components of the patient and observer scar assessment scale (POSAS) three months after surgery. At the same time, scar assessment was conducted by five observers (plastic surgeons) using the POSAS and Vancouver scar scale (VSS).

2.1. Patient and Observer Scar Assessment Scale. The POSAS is a scar assessment tool developed in 2004 and has both an observer and a patient scar rating system [8]. The observer scar assessment scale (OSAS) was rated through five components (vascularity, pigmentation, thickness, relief, and 
pliability) in the original version, and in the modified version, two components (surface area, overall opinion) were added and used [9]. The patient scar assessment scale (PSAS) was rated through 6 components (pain, itching, color, stiffness, thickness, and irregularity). Each component is rated with a 10-point scoring system, with a score of 1 for normal skin and 10 for the worst imaginable scar or sensation (Supplementary 1).

2.2. Vancouver Scar Scale. Introduced in 1990, the VSS was the first validated scar scale to be adopted in clinical practice for the assessment of burn scars and remains one of the most frequently used scales to date [10]. Scars are assessed based on four variables: pigmentation, vascularity, pliability, and height. Scores are then assigned across these four variables based on the degree of variance from normal skin (Supplementary 2).

2.3. Surgical Technique. First, the RSTL of the skin cancer site was marked. Wide excision for skin cancer was performed considering RSTL. The margin was determined between 3 and $6 \mathrm{~mm}$ considering the skin cancer type and patient risk. All defects were dissected into the subcutaneous layer. The donor site was selected as one of the posterior auricular area and the supraclavicular area considering the skin texture and color of the defect site. The RSTL of the donor site was then marked. In the RSTL-matched group, the graft was harvested at the donor site, trimmed according to the defect, and applied to facial defects in accordance with RSTL. In the RSTL-unmatched group, the harvested graft was fixed to the facial defect regardless of the RSTL of the donor graft. Finally, the graft was fixed in a tie-over manner using \#5-0 black silk. The donor site was closed primarily.

2.4. Statistical Analysis. First, the intraclass correlation coefficient (ICC) of the component of the VSS and observer component of the OSAS was measured to check the reliability between the five observers. In other literature, an ICC within the range of 0 to 0.20 was considered as "slight," 0.21 to 0.40 as "fair," 0.41 to 0.60 as "moderate," 0.61 to 0.80 as "substantial," and 0.81 to 1.0 as "almost perfect" [11]. The correlation between the patient component of the POSAS and the observer component of the VSS and the POSAS was evaluated using Pearson's correlation statistics.

The scar assessment scale difference between the groups that underwent FTSG according to RSTL and the group that underwent FTSG regardless of RSTL was analyzed using the Mann-Whitney $U$ test. Statistical significance was set at $p<0.05$. Statistical analyses were performed using PASW statistics 26 (IBM, Armonk, NY, USA; formerly SPSS statistics).

\section{Result}

From October 2013 to July 2019, 21 patients (5 males and 16 females) were included. The average age was 75.9 years. There were nine patients $(42.9 \%)$ in the RSTL-matched group and $12(57.1 \%)$ in the RSTL-unmatched group. The skin cancer types were basal cell carcinoma $(n=9)$, squamous cell carcinoma $(n=6)$, and Bowen's disease $(n=6)$.
TABLE 1: Characteristics of the patients.

\begin{tabular}{|c|c|}
\hline Characteristics & $n(\%)$ \\
\hline \multicolumn{2}{|l|}{ Sex } \\
\hline Male & $5(23.8)$ \\
\hline Female & $16(76.2)$ \\
\hline \multicolumn{2}{|l|}{ RSTL } \\
\hline Matched & $9(42.9)$ \\
\hline Unmatched & $12(57.1)$ \\
\hline \multicolumn{2}{|l|}{ Age (yr) } \\
\hline $40 \sim 49$ & $1(4.8)$ \\
\hline $50 \sim 59$ & $1(4.8)$ \\
\hline $60 \sim 69$ & $3(14.3)$ \\
\hline $70 \sim 79$ & $6(28.6)$ \\
\hline $80 \sim 89$ & $7(33.3)$ \\
\hline $90 \sim 99$ & $3(14.3)$ \\
\hline \multicolumn{2}{|l|}{ Defect area $\left(\mathrm{mm}^{2}\right)$} \\
\hline $100-199$ & $3(14.3)$ \\
\hline $200-299$ & $3(14.3)$ \\
\hline $300-399$ & $2(9.5)$ \\
\hline $400-499$ & $4(19.0)$ \\
\hline $500-599$ & $2(9.5)$ \\
\hline$>600$ & $7(33.3)$ \\
\hline \multicolumn{2}{|l|}{ Type of malignant skin cancer } \\
\hline Basal cell carcinoma & $9(42.9)$ \\
\hline Squamous cell carcinoma & $6(28.6)$ \\
\hline Bowen's disease & $6(28.6)$ \\
\hline \multicolumn{2}{|l|}{ Site of malignant skin tumor } \\
\hline Cheek & $6(28.6)$ \\
\hline Nose & $10(47.6)$ \\
\hline Philtrum & $1(4.8)$ \\
\hline Temple & $4(19.0)$ \\
\hline \multicolumn{2}{|l|}{ Location of skin donor site } \\
\hline Postauricular & $7(33.3)$ \\
\hline Supraclavicular & $14(66.7)$ \\
\hline
\end{tabular}

The defect sites were the cheek $(n=6)$, nose $(n=10)$, philtrum $(n=1)$, and temple $(n=4)$, while donor sites were postauricular $(n=7)$ and supraclavicular $(n=14)$ areas (Table 1).

3.1. Interobserver Reliability. Interobserver reliability was almost perfect in total (0.854) of the VSS and vascularity (0.822), overall (0.837), and total (0.823) of the observer component of the POSAS. In the VSS, pigmentation (0.568) was fair, and pliability (0.678) and height (0.637) were substantial. In the OSAS, pigmentation (0.787), thickness (0.686), relief (0.658), pliability (0.764), and surface area (0.781) were substantial (Table 2).

3.2. Correlation between the Patient Component of the POSAS (PSAS) and the Observer Component of the POSAS (OSAS) and VSS. Among the patient components of the POSAS (PSAS), color was matched with the VSS component 
TABLE 2: Interobserver reliability of the Vancouver scar scale (VSS) and the observer component of the patient and observer scar assessment scale (OSAS).

\begin{tabular}{lcc}
\hline Variable & Single measure ICC (95\% CI) & Average measure ICC (95\% CI) \\
\hline VSS & & $0.739(0.508 \sim 0.881)$ \\
Vascularity & $0.362(0.171 \sim 0.596)$ & $0.568(0.186 \sim 0.803)$ \\
Pigmentation & $0.208(0.044 \sim 0.449)$ & $0.678(0.393 \sim 0.8535)$ \\
Pliability & $0.296(0.115 \sim 0.537)$ & $0.637(0.316 \sim 0.834)$ \\
Height & $0.260(0.084 \sim 0.501)$ & $0.854(0.726 \sim 0.934)$ \\
Total & $0.540(0.346 \sim 0.738)$ & $0.822(0.664 \sim 0.919)$ \\
Observer component of the POSAS & & $0.787(0.598 \sim 0.903)$ \\
Vascularity & $0.480(0.283 \sim 0.693)$ & $0.686(0.408 \sim 0.857)$ \\
Pigmentation & $0.425(0.229 \sim 0.650)$ & $0.658(0.355 \sim 0.844)$ \\
Thickness & $0.304(0.121 \sim 0.544)$ & $0.764(0.555 \sim 0.892)$ \\
Relief & $0.278(0.099 \sim 0.519)$ & $0.781(0.587 \sim 0.900)$ \\
Pliability & $0.392(0.199 \sim 0.623)$ & $0.837(0.692 \sim 0.925)$ \\
Surface area & $0.416(0.222 \sim 0.643)$ & $0.823(0.666 \sim 0.919)$ \\
Overall & $0.506(0.310 \sim 0.713)$ & $0.482(0.285 \sim 0.694)$ \\
Total & & \\
\hline
\end{tabular}

TABLE 3: Correlation between the patient component of the POSAS (PSAS) and Vancouver scar scale (VSS) and observer component of the POSAS (OSAS).

\begin{tabular}{lcc}
\hline & Pearson's correlation coefficient & $p$ value \\
\hline VSS vascularity score vs. PSAS color score & -0.068 & 0.77 \\
VSS pigmentation score vs. PSAS color score & 0.038 & 0.87 \\
VSS pliability score vs. PSAS stiffness score & 0.657 & $0.001^{*}$ \\
VSS height score vs. PSAS thickness score & 0.542 & $0.011^{*}$ \\
VSS total score vs. PSAS total score & 0.708 & $\leq 0.001^{*}$ \\
OSAS vascularity score vs. PSAS color score & 0.168 & 0.465 \\
OSAS pigmentation score vs. PSAS color score & -0.081 & 0.727 \\
OSAS pliability score vs. PSAS stiffness score & 0.258 & 0.259 \\
OSAS thickness score vs. PSAS thickness score & 0.069 & 0.768 \\
OSAS relief score vs. PSAS irregularity score & 0.253 & 0.268 \\
OSAS overall score vs. PSAS overall score & 0.129 \\
OSAS total score vs. PSAS total score & 0.363 \\
\hline
\end{tabular}

of vascularity and pigmentation and with the POSAS observer component (OSAS) of vascularity and pigmentation. PSAS stiffness was matched with the VSS component of pliability and the OSAS component of pliability. PSAS thickness was matched with the VSS component of height and OSAS component of thickness; PSAS irregularity and overall were matched with OSAS component of relief and overall, respectively [12]. Among these, PSAS stiffness vs. VSS pliability, PSAS thickness vs. VSS height, and PSAS total vs. VSS total had significant results (Table 3).

3.3. VSS Comparison according to the RSTL. The RSTLmatched and RSTL-unmatched groups showed significant differences in all components of the VSS. The average VSS component of "total" in the RSTL-matched group was 1.78 and that in the RSTL-unmatched group was 3.98 , with a $p$ value of $\leq 0.001$. Similarly, the $p$ value of each component was significantly different from "pigmentation" (0.023), "vascularity" (0.007), "pliability" $(\leq 0.001)$, and "height" (0.049) (Table 4).

3.4. Observer Scar Assessment Scale Comparison according to RSTL. There were significant differences between the RSTL-matched and RSTL-unmatched groups in the OSAS component. The average OSAS component of "total" in the RSTL-matched group was 10.98 and that in the RSTLunmatched group was 14.49, and there was a significant difference with a $p$ value of 0.002 . In addition, significant differences were found in "pliability" (0.002), "surface area" (0.002), and "overall" (0.003). There were no significant differences in "vascularity" (0.464), "pigmentation" (0.082), “thickness" (0.148), and "relief” (0.095) (Table 5). 
TABLE 4: Vancouver scar scale comparison according to RSTL.

\begin{tabular}{lccccc}
\hline & RSTL matched (SD) & RSTL unmatched (SD) & Total (SD) & Mann-Whitney $U$ & $p$ value \\
\hline Pigmentation & $0.42(0.35)$ & $0.85(0.4)$ & $0.67(0.43)$ & 85.5 & $0.023^{*}$ \\
Vascularity & $0.38(0.46)$ & $0.97(0.35)$ & $0.71(0.49)$ & 91 & $0.007^{*}$ \\
Pliability & $0.51(0.32)$ & $1.3(0.43)$ & $0.96(0.55)$ & 101 & $\leq 0.001^{*}$ \\
Height & $0.47(0.49)$ & $0.87(0.39)$ & $0.7(0.47)$ & 81.5 & $0.049^{*}$ \\
Total & $1.78(1.12)$ & $3.98(0.99)$ & $3.04(1.52)$ & 101 & $\leq 0.001^{*}$ \\
\hline
\end{tabular}

TABLE 5: Observer scar assessment scale comparison according to RSTL.

\begin{tabular}{|c|c|c|c|c|c|}
\hline & RSTL matched (SD) & RSTL unmatched (SD) & Total (SD) & Mann-Whitney $U$ & $p$ value \\
\hline Vascularity & $1.40(0.48)$ & $1.95(1.29)$ & $1.71(1.04)$ & 65 & 0.464 \\
\hline Pigmentation & $1.62(0.61)$ & $2.71(2.25)$ & $2.25(1.22)$ & 78.5 & 0.082 \\
\hline Thickness & $1.58(0.70)$ & $2.03(0.84)$ & $1.84(0.80)$ & 74.5 & 0.148 \\
\hline Relief & $1.56(0.43)$ & $2.12(0.75)$ & $1.88(0.68)$ & 78 & 0.095 \\
\hline Pliability & $1.56(0.41)$ & $2.48(0.68)$ & $2.01(0.74)$ & 94.5 & $0.002^{*}$ \\
\hline Surface area & $1.58(0.65)$ & $2.97(1.07)$ & $2.37(1.14)$ & 95.5 & $0.002^{*}$ \\
\hline Overall & $1.69(0.70)$ & $2.85(1.00)$ & $2.35(1.05)$ & 94 & $0.003^{*}$ \\
\hline Total & $10.98(2.98)$ & $17.12(5.48)$ & $14.49(5.46)$ & 95 & $0.002^{*}$ \\
\hline
\end{tabular}

Mann-Whitney $U$ test.

TABLe 6: Patient scar assessment scale comparison according to RSTL.

\begin{tabular}{|c|c|c|c|c|c|}
\hline & RSTL matched (SD) & RSTL unmatched (SD) & Total (SD) & Mann-Whitney $U$ & $p$ value \\
\hline Painful & $1(0)$ & $1.42(0.51)$ & $1.24(0.44)$ & 76.5 & 0.111 \\
\hline Itching & $1.22(0.67)$ & $1.25(0.45)$ & $1.24(0.54)$ & 60 & 0.702 \\
\hline Color & $1.78(0.83)$ & $1.83(0.83)$ & $1.81(0.81)$ & 56 & 0.917 \\
\hline Stiffness & $1(0)$ & $1.92(0.79)$ & $1.52(0.75)$ & 90 & $0.009^{*}$ \\
\hline Thickness & $1.11(0.33)$ & $2.67(0.98)$ & $2(1.1)$ & 100.5 & $\leq 0.001^{*}$ \\
\hline Irregularity & $1.44(0.73)$ & $2.58(0.79)$ & $2.1(0.94)$ & 91 & $0.007^{*}$ \\
\hline Overall & $1.33(0.5)$ & $2.25(0.97)$ & $1.86(0.91)$ & 88.5 & $0.012^{*}$ \\
\hline Total & $8.89(1.54)$ & $13.92(2.02)$ & $11.76(3.11)$ & 108 & $\leq 0.001^{*}$ \\
\hline
\end{tabular}

Mann-Whitney $U$ test.

3.5. Patient Scar Assessment Scale (PSAS) Comparison according to RSTL. There were significant differences between the RSTL-matched and RSTL-unmatched groups in the PSAS component. The average of the PSAS component of "total" in the RSTL-matched group was 8.89 and that in the RSTL-unmatched group was 13.92, with a significant difference $(\leq 0.001)$. In addition, significant differences were found in "stiffness" $(p=0.009)$, "thickness" $(p \leq 0.001)$, "irregularity" ( $p=0.007)$, and "overall" $(p=0.012)$. There were no significant differences in "painful" $(p=0.111)$, “itching" ( $p=0.702)$, and "color" $(p=0.917)$ (Table 6).

\section{Discussion}

FTSG can be a simple and esthetic reconstruction option when used correctly. FTSG has a short learning course and can be performed without any burden. In addition, because it does not distort the surrounding structures, the surrounding tissue can be preserved for later [2]. However, fullthickness skin may look outstanding due to a mismatch of skin color and texture of donor and recipient sites. To reduce this mismatch of the grafted skin, surgeons use donor sites with similar skin color and texture, which is essential in static conditions. Since the skin repeatedly stretches and contracts according to the movement, we suspect that matching the dynamic component by matching the RSTL would make the result better. It was also suggested that if the dynamic components were matched, there would be a significant difference between wound healing and marginal scar formation.

According to previous literature, RSTL shows a parallel arrangement of collagen bundles of the dermal layer when intrinsic tension is made $[13,14]$. In order to avoid a possibly disordered arrangement of collagen fibers in the scarring 


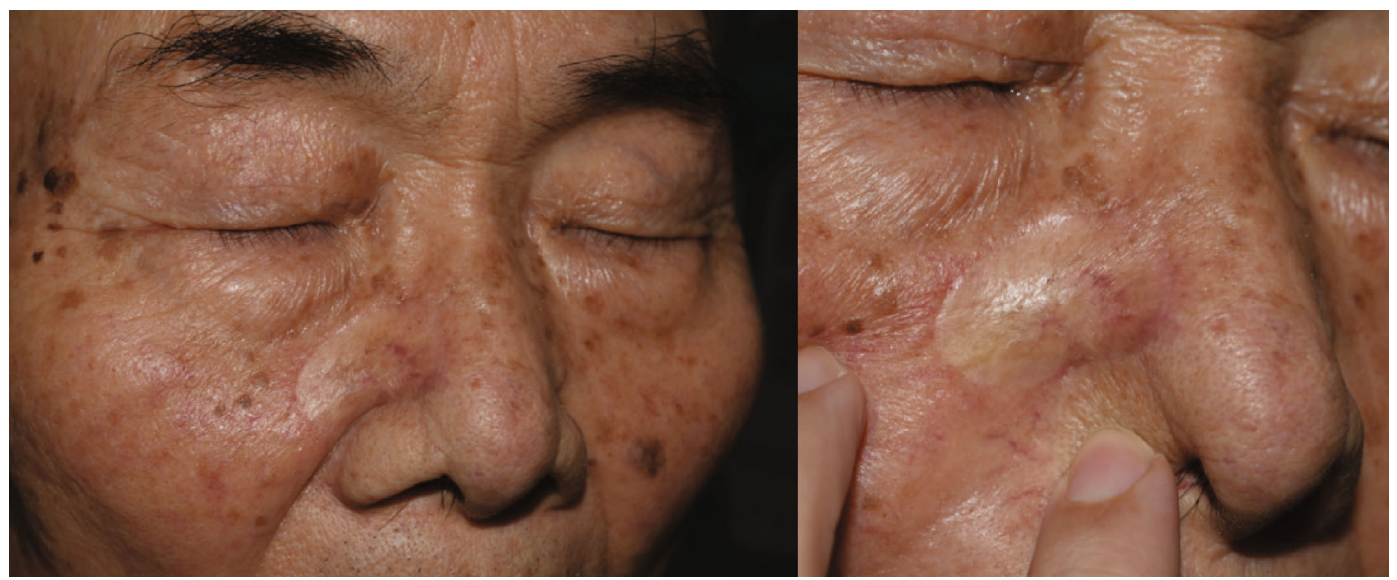

FIGURE 2: Photograph taken three months after the RSTL-matched FTSG. The patient underwent a FTSG according to RSTL after wide excision for BCC on the right cheek. The patient gave 1 point for the "thickness," and the score was lower than the overall average (2.0). However, the observer gave 2 points for the "thickness," and the score was higher than the average (1.84), and the observer gave 1 point for the "surface area," and the score was lower than the average (2.37). The observer component of "thickness" was not significant, and "surface area" was significant. These two observer components are expressed as a single component called "thickness" to the patient. Therefore, this resulted in the patient's "thickness" being accepted as a meaningful result.

wound [15], these collagen bundles must be aligned in the same direction to reduce scarring.

In the VSS comparison according to RSTL, the RSTLmatched and unmatched groups showed significant differences in all components, whereas in the OSAS comparison according to RSTL, there were only significant differences in four components: "pliability," "surface area," "overall," and "total." Unlike all components in OSAS that were graded on a ten-point scale, the VSS is represented by 0 to 3 points for "vascularity," 0 to 2 points for "pigmentation," 0 to 5 points for "pliability," and 0 to 3 points for "height." In addition, the VSS has 4 components, and the OSAS has 7 components. In fact, "vascularity," "pigmentation," and "thickness," which had no significant difference in OSAS, were expressed as 0 to 2 points and 0 to 3 points in the VSS, so the interval between scores can be said to be relatively large compared to those in the OSAS.

In the PSAS comparison according to RSTL, there were significant differences in four components: "stiffness," "thickness," "irregularity," and "overall." This should be considered in comparison to the results of the OSAS comparison according to RSTL. PSAS stiffness can be matched to OSAS pliability. PSAS irregularity can be matched to OSAS relief, and overall PSAS can be matched to overall OSAS [12]. However, the thickness, which had a significant difference in the patient (Table 6), was not significant in the observer (Table 5). The reason may be the thickness of the patient reflects a mixture of thickness and evaluation of the surface area when touched. Indeed, the evaluation of the surface area of the observer was significant. For example, Figure 2 shows a picture of a patient who underwent a FTSG according to RSTL after wide excision for BCC on the right cheek. The patient gave 1 point for the "thickness," and the score was lower than the overall average (2.0). However, the observer gave 2 points for the "thickness" and the score was higher than the average (1.84), and the observer gave 1 point for the "surface area" and the score was lower than the average (2.37). When the patient's "thickness" included the observer's "thickness" and the "surface area," the "thickness" was not significant in the observer, but the "surface area" was significant. This resulted in the patient's "thickness" being accepted as a meaningful result.

It is very difficult to obtain an esthetic outcome due to dimpling scar formation when performing graft in deep wounds, and in this case, the local flap could often be more meaningful [16]. Also, local flaps may be considered the reconstructive option for small- to moderate-sized defects of the face [17-19]. Although a FTSG is difficult to secure sufficient thickness and requires a well-vascularized wound bed with intact underlying structures, if the graft that was performed on the wound bed met these conditions, FTSG could be a good reconstruction option of the facial defect. We limited FTSG to facial skin cancer in this study, since skin cancer generally does not injure the underlying musculature when performing wide excision. From the patient's point of view, some patients felt a lot of pressure about the increase in the surgical range and the length of the scar when the local flap was applied. In the case of using FTSG, even if skin color discrepancy occurred immediately after surgery, the difference was reduced over time, so the satisfaction was high.

The RSTL-matched group showed a natural appearance with surrounding tissue in the dynamic animation phase compared to the unmatched group. Figure 3 shows a patient who underwent wide excision for basal cell carcinoma located in the left lateral canthal area and RSTL-matched FTSG. In the closed-eye view, wrinkles on the graft site are shown in harmony with the surrounding tissue. However, Figure 4 is a patient who underwent wide excision of basal cell carcinoma located in the infraorbital area and RSTLunmatched FTSG. In the static phase, the graft site does not look prominent, but in the closed-eye view, the wrinkles do not appear when compared to the contralateral side, so the graft site had a "surgical" appearance. We suspected that 


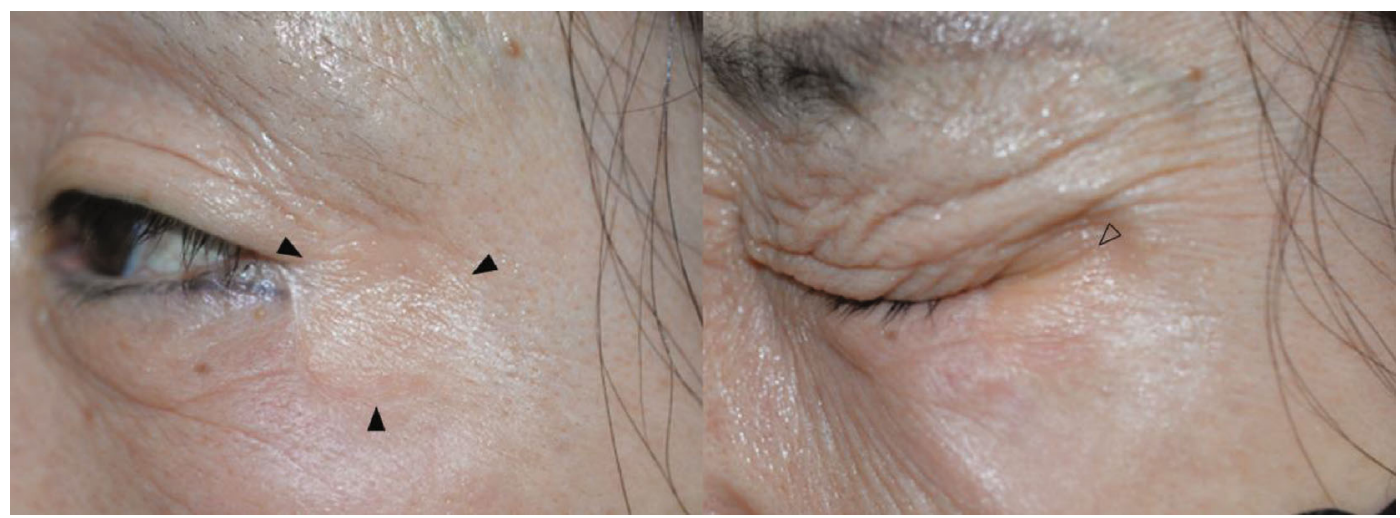

FIGURE 3: Photograph taken two years after the RSTL-matched FTSG. Black arrow indicates graft site. The empty arrow shows natural wrinkles during facial animation.

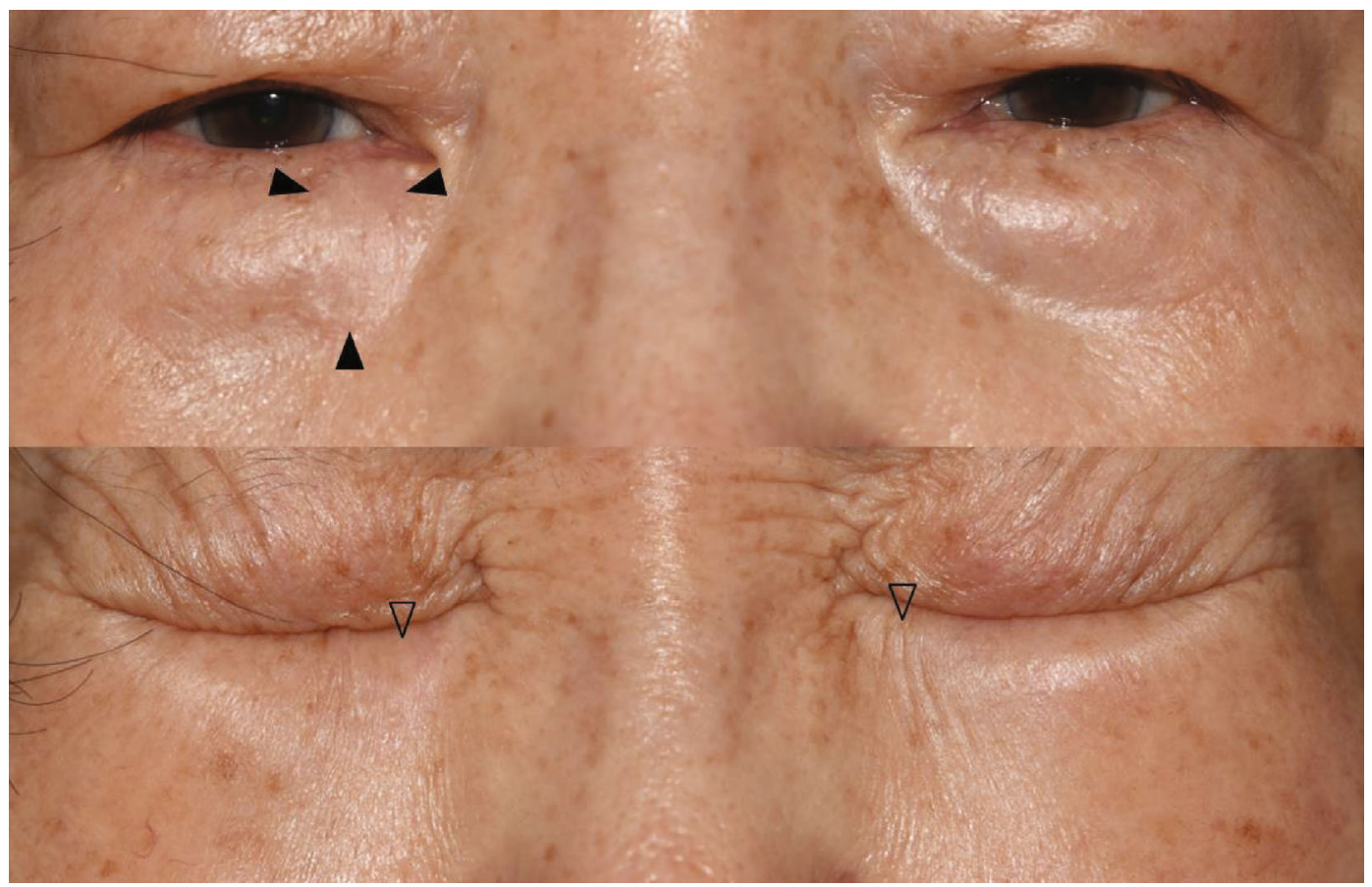

FIGURE 4: Photograph taken two years after the RSTL-unmatched FTSG. Black arrow indicates graft site. When comparing the empty arrows on both sides, the graft site does not clearly show wrinkle formation.

the RSTL-matched FTSG was effective in the dynamic phase when the skin was thin and the musculature was close to the wound bed.

We have not demonstrated how much better esthetic and functional results the RTSL-matched FTSG can achieve with facial musculature motion in this study. This is because there has been no discussion on the dynamic scar assessment scale. However, the authors were able to identify natural textures in the RSTL-matched group during facial animation (Figure 3). We believe this is because collagen fibers are arranged in a relatively parallel manner.

Figure 5 shows patients undergoing FTSG with wide excision for basal cell carcinoma located on the right nasal sidewall. Patients were followed up three years after surgery. Figure 5(a) presents a patient who has undergone RSTL- unmatched FTSG, and Figure 5(b) illustrates a patient who has undergone RSTL-matched FTSG. Compared to RSTLunmatched FTSG, the contour and texture of the RSTLmatched graft are naturally seen with surrounding tissues. Statistical effectiveness could not be demonstrated due to the lack of follow-up observations on sufficient patients, but the author suspected that as time passed and scar maturation occurred, the effect of RSTL-matched graft would be excellent.

Although we successfully covered facial defects with RSTL-matched FTSG, the present study has some limitations to acknowledge. First, it included a small number of cases and used a nonrandomized retrospective design. Thus, selection biases and the presence of confounding factors are unavoidable. Second, further discussion is needed to use the 


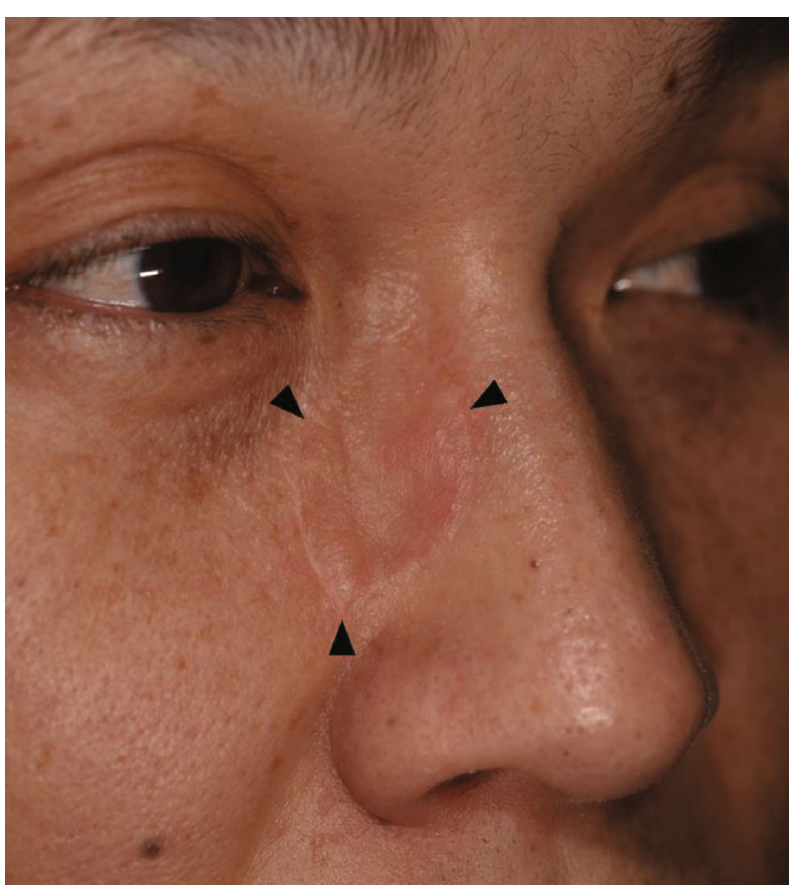

(a)

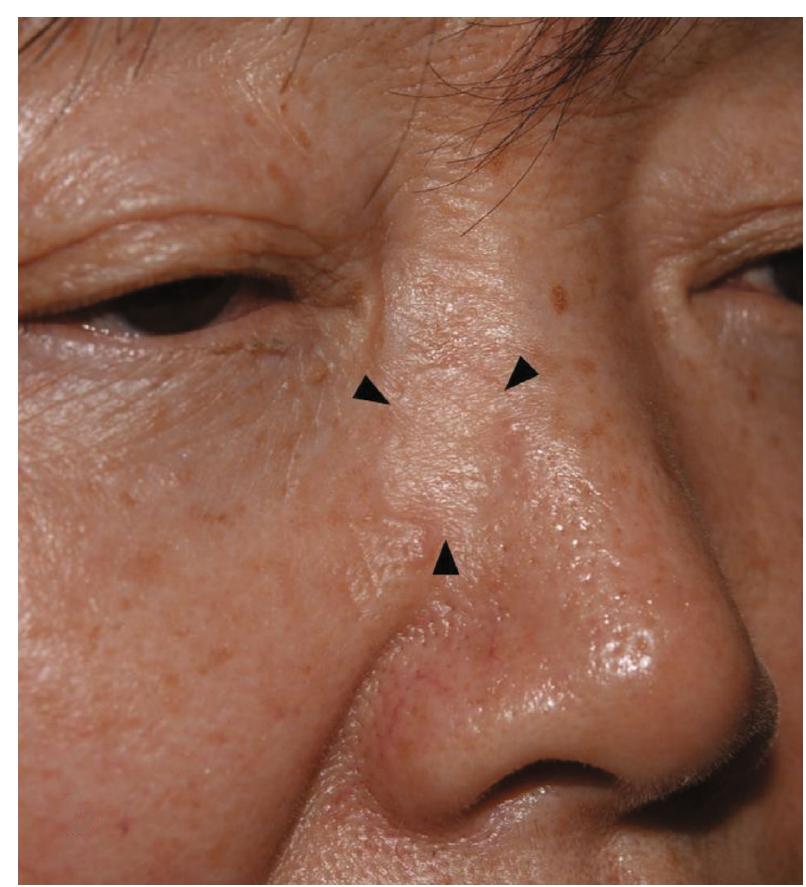

(b)

FIGURE 5: (a) Photograph of RSTL-unmatched patient taken three years postoperatively and (b) photograph of RSTL-matched patient taken three years postoperatively. Compared to the RSTL-unmatched patient, the RSTL-matched patient shows a more natural look with the surrounding tissue.

FTSG according to RSTL for areas other than the face. RSTL has many definitions in previous literature [20,21]. Various skin lines, such as Cox's line and Rubin's line, are defined, including the most frequently mentioned Langer's line. In the face, skin lines are mostly defined similarly, but those of the trunk and extremity are defined differently according to motion and position [22]. This is because tension acts differently depending on the position. Therefore, the author believes that skin grafts according to RSTL can be applied consistently to faces defined by similar skin tension lines.

\section{Conclusion}

A FTSG can be an attractive option when superficial defects are present on the face. When FTSG was performed, matching RSTL in the facial area showed significant scar quality improvement. Although the evaluation method was limited, RSTL-matched FTSG showed a natural appearance due to better assimilation to facial animation. Therefore, when covering superficial defects on the face with FTSG, matching the graft's RSTL along the surrounding RSTL can result in better scar quality and facial esthetics.

\section{Data Availability}

The datasets and/or analysis results used during the current study are available from the corresponding authors upon reasonable request.

\section{Additional Points}

Key Messages. (i) A FTSG can be an attractive option when superficial defects are present on the face. (ii) When FTSG was performed, matching RSTL in the facial area could provide significant scar quality improvement.

\section{Disclosure}

An earlier version of this paper has been presented at the International Conference on PRS Korea 2020.

\section{Conflicts of Interest}

All authors declare they have no conflicts of interest.

\section{Supplementary Materials}

Supplementary 1: patient and observer scar assessment scale. Supplementary 2: Vancouver scar scale. (Supplementary Materials)

\section{References}

[1] C. S. Yoon, S. I. Kim, H. Kim, and K. N. Kim, "Keystonedesigned perforator island flaps for the coverage of traumatic pretibial defects in patients with comorbidities," The International Journal of Lower Extremity Wounds, vol. 16, no. 4, pp. 302-309, 2017.

[2] G. Pietramaggiori, S. S. Scherer-Pietramaggiori, and D. P. Orgill, "Skin grafting and dermal substitute placement," in 
Operative Procedures in Plastic, Aesthetic and Reconstructive Surgery, CRC Press, 2015.

[3] T. M. Johnson, D. Ratner, and B. R. Nelson, "Soft tissue reconstruction with skin grafting," Journal of the American Academy of Dermatology, vol. 27, no. 2, pp. 151-165, 1992.

[4] E. C. Padgeit and K. L. Stephenson, "Calibrated intermediate skin grafts," Plastic and Reconstructive Surgery, vol. 39, no. 2, pp. 195-209, 1967.

[5] A. Stephenson, R. Griffiths, and T. La Hausse-Brown, "Patterns of contraction in human full thickness skin grafts," British journal of plastic surgery, vol. 53, no. 5, pp. 397-402, 2000.

[6] O. F. Osman and S. Emara, "Extended use of full-thickness skin grafts, employing variable donor sites," World journal of plastic surgery, vol. 7, no. 2, pp. 159-165, 2018.

[7] A. F. Borges and J. E. Alexander, "Relaxed skin tension lines, Z-plasties on scars, and fusiform excision of lesions," British journal of plastic surgery, vol. 15, pp. 242-254, 1962.

[8] L. J. Draaijers, F. R. Tempelman, Y. A. Botman et al., “The patient and observer scar assessment scale: a reliable and feasible tool for scar evaluation," Plastic and reconstructive Surgery, vol. 113, no. 7, pp. 1960-1965, 2004.

[9] A. L. van de Kar, L. U. Corion, M. J. Smeulders, L. J. Draaijers, C. M. van der Horst, and P. P. van Zuijlen, "Reliable and feasible evaluation of linear scars by the patient and observer scar assessment scale," Plastic and reconstructive surgery, vol. 116, no. 2, pp. 514-522, 2005.

[10] T. Sullivan, J. Smith, J. Kermode, E. Mclver, and D. Courtemanche, "Rating the burn scar," The Journal of burn care \& rehabilitation, vol. 11, no. 3, pp. 256-260, 1990.

[11] J. R. Landis and G. G. Koch, "The measurement of observer agreement for categorical data," Biometrics, vol. 33, no. 1, pp. 159-174, 1977.

[12] J. K. Chae, J. H. Kim, E. J. Kim, and K. Park, "Values of a patient and observer scar assessment scale to evaluate the facial skin graft scar," Annals of Dermatology, vol. 28, no. 5, pp. 615$623,2016$.

[13] I. Brown, "Scanning electron microscopy of human dermal fibrous tissue," Journal of Anatomy, vol. 113, Part 2, pp. 159168, 1972.

[14] G. E. Piérard and C. M. Lapière, "Microanatomy of the dermis in relation to relaxed skin tension lines and Langer's lines," The American journal of dermatopathology, vol. 9, no. 3, pp. 219224, 1987.

[15] P. D. Verhaegen, P. P. M. van Zuijlen, N. M. Pennings et al., "Differences in collagen architecture between keloid, hypertrophic scar, normotrophic scar, and normal skin: an objective histopathological analysis," Wound Repair and Regeneration, vol. 17, no. 5, pp. 649-656, 2009.

[16] K. S. Lee, J. O. Kim, N. G. Kim, Y. J. Lee, Y. J. Park, and J. S. Kim, "A comparison of the local flap and skin graft by location of face in reconstruction after resection of facial skin cancer," Archives of craniofacial surgery, vol. 18, no. 4, pp. 255-260, 2017.

[17] C. S. Yoon, H. B. Kim, Y. K. Kim, H. Kim, and K. N. Kim, "Relaxed skin tension line-oriented keystone-designed perforator island flaps considering the facial aesthetic unit concept for the coverage of small to moderate facial defects," Medicine, vol. 98, no. 3, article e14167, 2019.

[18] S. Y. Lim, C. S. Yoon, H. G. Lee, and K. N. Kim, "Keystone design perforator island flap in facial defect reconstruction,"
World Journal of Clinical Cases, vol. 8, no. 10, pp. 1832$1847,2020$.

[19] H. G. Lee, Y. T. Kong, and K. N. Kim, "Use of Keystone Flaps in Consideration of the Facial Aesthetic Subunit Concept as an Alternative Reconstructive Option for Nasal Defect Coverage," Journal of Craniofacial Surgery, vol. 32, no. 5, pp. 1864-1869, 2021.

[20] A. F. Borges, "Relaxed skin tension lines (RSTL) versus other skin lines," Plastic and reconstructive surgery, vol. 73, no. 1, pp. 144-150, 1984.

[21] B. J. Wilhelmi, S. J. Blackwell, and L. G. Phillips, "Langer's lines: to use or not to use," Plastic and reconstructive surgery, vol. 104, no. 1, pp. 208-214, 1999.

[22] S. P. Paul, "Biodynamic excisional skin tension lines for excisional surgery of the lower limb and the technique of using parallel relaxing incisions to further reduce wound tension," Plastic and Reconstructive Surgery - Global Open, vol. 5, no. 12, article e1614, 2017. 